\title{
Application of two dimensional thin layer chromatography pattern comparison for fingerprinting the active compounds in the leaves of Vitex trifolia Linn possessing anti- tracheospasmolytic activity.
}

\begin{abstract}
We have developed one approach to fingerprint and estimate the active compounds in the leaves of Vitex trifolia Linn possessing anti-tracheospasmolytic assay using two-dimensional TLC pattern comparison. Based on the two-dimensional TLC pattern and the activity of the centrifugal partition chromatography fractions, we concluded that the semi polar compounds were responsible for anti-tracheospasmolytic activity. The best non-polar/semi polar mobile phases for the two-dimensional TLC using silica gel as the stationary phase were chloroform/methanol (9/1) as the first mobile phase and ethyl acetate/chloroform/methanol $(28 / 28 / 44)$ as the second mobile phase.
\end{abstract}

Keyword: Asthma; Centrifugal partition chromatography; Fingerprinting; Thin layer chromatography; Tracheospasmolytic assay; Vitex trifolia Linn. 